\title{
Developing a Generic Agent-based Intelligent Tutoring System
}

\author{
M. Hospers, E. Kroezen, A. Nijholt, R. op den Akker \& D. Heylen \\ Center of Telematics and Information Technology \\ University of Twente, Enschede, the Netherlands \\ anijholt@,cs.utwente.nl
}

\begin{abstract}
We developed a generic teaching environment that uses agents to support learning. An agent platform developed in our research group is used. The system is generic in the sense that the cognition core (domain model, student model and instruction model) is separated from the exercise modules and user interfaces. The architecture allows different user interfaces. An application of the environment for nurse training has been implemented.
\end{abstract}

\section{Introduction}

We developed an agent-based generic system (INES) where we have a clear separation between the concrete user interface, the abstract user interface and the cognition core of the system [2]. A number of agents have been implemented. They observe the students actions and check whether they are performed correctly and in a correct order. The agents are capable of providing feedback, explanations and demonstrations. Exercises typically consist of subtasks with some partial ordering defined for them. They are provided to the system using XML files and they can be filled in for repairing a flat tire as easy as for a nursing task. Different user interfaces can be added to the system without changing the agent-based core. The implemented system has been applied to a nurse training task. An exercise file and a simple interface were made for the subcutaneous injection (see Figure 1).

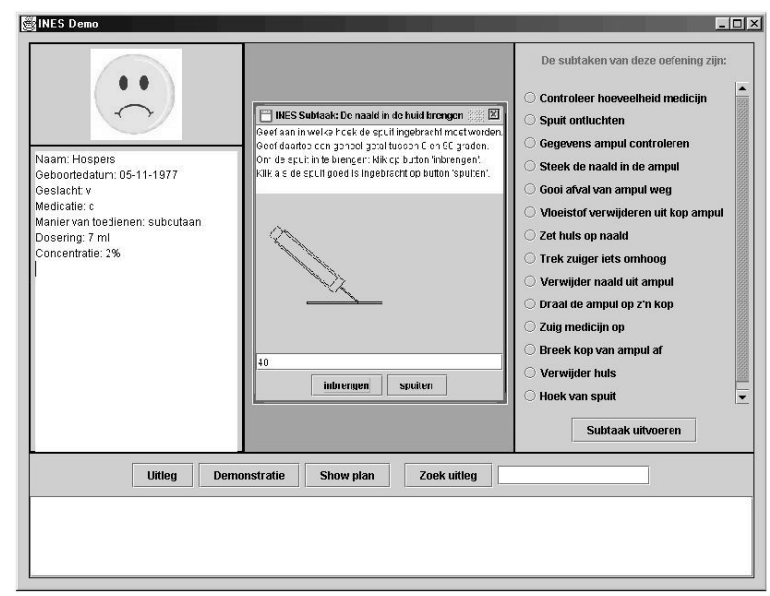

Figure 1. User interface for nurse training

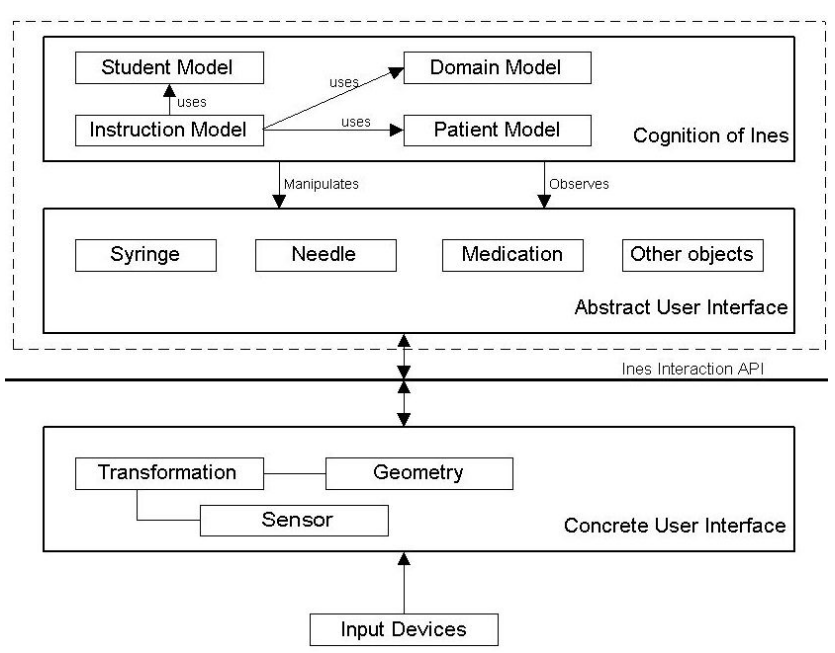

Figure 2. The system architecture

\section{System Architecture and Cognition Core}

The architecture of the system is shown in Figure 2. The cognition core consists of four modules: the domain model, the student model, the instruction model and, for our application, the patient model that has been added to the system. The domain model has to be filled with the exercises (including explanations and demonstration) in XML files. In the cognition core we find proactive and reactive agents that observe the student and provide feedback. Agents can be added to the system. In our nursing application we introduced a proactive agent for checking the sterility of the objects used by the nurse.

\section{Future Research}

Several small-sized projects to extend the system and other agent-based educational environments (e.g. [1]) are in development for further study of our approach. The extensions include the introduction of a virtual reality environment and the use of haptic devices in the interface.

\section{References}

1. Broersen, A. \& A. Nijholt. Developing a Virtual Piano Playing Environment. In: Proc. ICALT 2002, 278-282.

2. Hospers, M., E. Kroezen, A. Nijholt. R. op den Akker \& D. Heylen. An Agent-based Intelligent Tutoring System for Nurse Education. CTIT Report, University of Twente, 2003. 